\title{
Associação de SARS-COV-2 com a doença de Kawasaki
}

\author{
Association of SARS-COV-2 with Kawasaki disease
}

\author{
Asociación del SARS-COV-2 con la enfermedad de Kawasaki
}

\begin{abstract}
Amanda Rodrigues Lessa ${ }^{1 *}$, Ana Carolina Rocha Auer ${ }^{1}$, Emmyli Nunes de Freitas ${ }^{1}$, Guilherme Rodrigues Lessa ${ }^{1}$, Igor Lobato de Assis Paula e Silva ${ }^{1}$, Rhainer Victor Simmer Schunk ${ }^{1}$, Rhaiane Evelyn Simmer Schunk ${ }^{1}$, Waltair Mota Filho ${ }^{1}$, Leonardo Soares Prates ${ }^{2}$.
\end{abstract}

\section{RESUMO}

Objetivo: Esta revisão bibliográfica buscou estabelecer uma associação entre a Doença de Kawasaki e a Covid-19 em crianças por meio das melhores evidências científicas até o momento sobre o assunto. Revisão bibliográfica: A Doença de Kawasaki (DK) é uma vasculite aguda, autolimitada e multissistêmica, podendo acometer vários órgãos, sendo o comprometimento cardíaco o mais significativo. Também é a causa mais comum de doença cardíaca adquirida em crianças em países desenvolvidos. Recentemente, relatos têm descrito uma associação, concordante com literaturas de base, entre DK e resposta hiper inflamatória da COVID-19 com acometimento multissistêmico, mas com uma média de idade superior em comparação com a DK clássica. Visto que os critérios diagnósticos para DK não fecham, alguns autores sugeriram a Síndrome Inflamatória Multissistêmica em Crianças (SIM-C) como a mais provável na associação à Covid-19. Considerações finais: Entende-se, portanto, que há necessidade de um tempo maior de observação epidemiológica da Covid-19 a fim de que se possa firmar uma associação entre ela e a doença de Kawasaki.

Palavras-chave: COVID-19, Doença de Kawasaki, Saúde da criança.

\begin{abstract}
Objective: This bibliographic review sought to establish an association between Kawasaki's disease and Covid-19 in children through the best scientific evidence to date on the subject. Literature review: Kawasaki disease (KD) is an acute, self-limited and multisystemic vasculitis, which can affect several organs, with cardiac involvement being the most significant. It is also the most common cause of heart disease acquired in children in developed countries. Recently, reports have described an association, in agreement with basic literature, between DK and COVID-19 hyperinflammatory response with multisystemic involvement, but with a higher average age compared to classic DK. Since the diagnostic criteria for KD do not close, some authors have suggested the Multisystemic Inflammatory Syndrome in Children (SIM-C) as the most likely in association with Covid-19. Final considerations: It is understood, therefore, that there is a need for a longer period of epidemiological observation of Covid-19 in order to establish an association between it and Kawasaki's disease.
\end{abstract}

Key words: COVID-19, Kawasaki disease, Child health.

\section{RESUMEN}

Objetivo: Esta revisión bibliográfica buscó establecer una asociación entre la enfermedad de Kawasaki y Covid-19 en niños a través de la mejor evidencia científica hasta la fecha sobre el tema. Revisión bibliográfica: La enfermedad de Kawasaki (EK) es una vasculitis aguda, autolimitada y multisistémica, que

\footnotetext{
${ }^{1}$ Universidade de Vila Velha (UVV), Vila Velha - ES. *E-mail: amanda.r-|@hotmail.com
}

${ }^{2}$ Universidade Federal de Juiz de Fora, Juiz de Fora - MG. 
puede afectar a varios órganos, siendo la afectación cardíaca la más significativa. También es la causa más común de enfermedad cardíaca adquirida en niños en países desarrollados. Recientemente, reportes han descrito una asociación, en concordancia con la literatura básica, entre la respuesta hiperinflamatoria DK y COVID-19 con afectación multisistémica, pero con una edad promedio superior a la DK clásica. Dado que los criterios diagnósticos para la EK no se cierran, algunos autores han sugerido que el Síndrome Inflamatorio Multisistémico en Niños (SIM-C) es el más probable asociado con Covid-19. Consideraciones finales: Por tanto, se entiende que es necesario un período más prolongado de observación epidemiológica de Covid-19 para establecer una asociación entre éste y la enfermedad de Kawasaki.

Palabras clave: COVID-19, La enfermedad de Kawasaki, Salud de los niños.

\section{INTRODUÇÃO}

A Doença de Kawasaki (DK) é uma vasculite aguda, autolimitada e multissistêmica, podendo acometer vários órgãos como o coração, sistema nervoso central, intestino, pulmão, entre outros. Por se tratar de uma vasculite de vasos de médio e pequeno calibre, e uma predileção pelas artérias coronárias, 0 comprometimento cardíaco é o mais significativo e a complicação mais temida, com o desenvolvimento de aneurismas de artérias coronárias em aproximadamente $25 \%$ dos casos não tratados, além de oclusão trombótica arterial ou, potencialmente, morte súbita, sendo, portanto, a causa mais comum de doença cardíaca adquirida em crianças em países desenvolvidos (MCCRINDLE BW, et al., 2017).

É uma das vasculites mais comuns na infância, perdendo apenas para a púrpura de Henoch-Schönlein vasculite mediada por IgA (YOUNGER DS, 2019). Apresenta uma incidência em todas as faixas etárias pediátricas, embora seja mais prevalente em meninos e em crianças menores de 5 anos, com raro acometimento em bebês com menos de 6 meses ou mais de 8 anos. Apesar de sua distribuição universal, afeta principalmente a população asiática e seus descendentes distribuídos pelo mundo (MCCRINDLE BW, et al., 2017; AGARWAL S e AGRAWAL DK, 2017). Sua forma clássica caracteriza-se pela presença de febre que perdura cinco dias ou mais, associada a pelo menos uma das seguintes manifestações: conjuntivite não exsudativa, alterações nos lábios ou cavidade oral, linfadenopatia cervical, erupção cutânea polimórfica, alterações nas extremidades na fase aguda ou subaguda (SANTOS BS, et al., 2020).

Um estudo realizado em 2010 no Japão, revelou uma incidência entre crianças menores de 5 anos de aproximadamente 215-240 casos por 100.000 habitantes por ano, entre 2007 e 2008, afetando majoritariamente meninos entre 6 a 11 meses, tornando o Japão o país com a maior incidência de DK no mundo, com aproximadamente 1 caso a cada 100 crianças menores de 5 anos. Outros países asiáticos também apresentaram incidência aumentada (NAKAMURA Y, et al., 2010).

Caracteriza-se por febre prolongada (maior ou igual a 5 dias), conjuntivite bilateral não exsudativa, eritema e edema de língua, lábios e mucosa oral, alterações de extremidades, linfonodomegalia cervical, exantema polimórfico e com uma história natural da doença de, aproximadamente, 12 dias (MCCRINDLE BW, et al., 2017).

O diagnóstico é essencialmente clínico, baseado nos critérios definidos em 2004 pela American Heart Association, que são a presença de febre com duração igual ou superior a cinco dias, associada a pelo menos quatro dos cinco seguintes critérios: Alterações nas extremidades (eritema palmo-plantar com ou sem edema doloroso ou descamação periungueal), Exantema polimorfo, Hiperemia conjuntival bulbar bilateral não exsudativa, Alterações dos lábios e da mucosa oral (eritema, fissuras, descamação) e linfadenopatia cervical (diâmetro superior a $1,5 \mathrm{~cm}$, geralmente unilateral, dura e indolor à palpação). Não apresenta teste diagnóstico/marcadores laboratoriais específicos, porém é altamente responsiva ao tratamento com imunoglobulina e aspirina, conduzindo à melhora clínica e reduzindo efetivamente as complicações cardíacas de $25 \%$ para $4,7 \%$, se tratado precocemente (BURNS JC e GLODÉ MP, 2004).

Sua etiologia permanece desconhecida, porém existem diversas teorias sobre as possíveis etiologias da DK, como fatores ambientais, agentes virais e agentes bacterianos (com confirmação de infecção prévia ao 
aparecimento de DK), parentesco (associação de membros da família do caso índice), predisposição genética (desfecho de DK em indivíduos geneticamente susceptíveis, secundário à infecção por um ou mais agentes que levariam a uma condição assintomática ou avasculares em indivíduos sem susceptibilidade), entre diversas outras teorias (BENSELER SM, et al., 2005; MCCRINDLE BW, et al., 2017; YOUNGER DS, 2019; BURGNER D e HARNDEN A, 2005; SUNDEL MDR, 2020).

Dentre os agentes virais descritos, pode-se destacar o SARS-COV-2, identificado inicialmente em Wuhan (província de Hubei, China) no fim de 2019, responsável pela doença COVID-19, reconhecida pela Organização Mundial da Saúde (OMS) em fevereiro de 2020. Por conta do aumento do número de casos globalmente, em março de 2020 foi declarado estado de pandemia por COVID-19 (ESPER F, et al., 2005).

Em abril de 2020, segundo Riphagen S, et al. (2020), o Reino Unido relatou uma apresentação similar à DK incompleta e a síndrome do choque tóxico e posteriormente outros países relataram diversos casos semelhantes, sendo denominados como Síndrome Inflamatória Multissistêmica em Crianças (SIM-C), também conhecida como Síndrome Inflamatória Multissistêmica Pediátrica, temporariamente associado com SARS-COV-2 (SIMP-TS) ou choque inflamatório pediátrico. Esses estudos mostraram a presença de resposta hiper inflamatória secundária ao SARS-COV-2, causando alterações coronarianas em crianças, semelhante à DK (LICCIADI F, et al., 2020; VERDONI L, et al., 2020).

Dada a importância do tema e a relevância dos últimos acontecimentos causados pela pandemia, a presente revisão bibliográfica busca apresentar as melhores evidências científicas disponíveis até o momento, que trazem uma associação entre DK e COVID-19 em crianças.

\section{REVISÃO BIBLIOGRÁFICA}

\section{Associação entre COVID-19 e a DOENÇA DE KAWASAKI}

Recentes relatos têm descrito uma associação, concordante com literaturas de base, entre DK e resposta hiper inflamatória do COVID-19 com acometimento multissistêmico. A DK afeta, na maioria das vezes, crianças menores de cinco anos, porém evidencia-se uma média de idade superior na associação da doença com COVID-19. Sugere-se que a família do coronavírus pode representar um dos gatilhos para DK, sendo a. SARS-CoV-2, uma cepa altamente virulenta, causando intensa resposta imune no hospedeiro (SANTOS BS, et al., 2020; VERDONI L, et al., 2020). Na associação entre Covid-19 e DK, o sistema imunológico inato é ativado, ocorrendo inflamação excessiva e danos sistêmicos. O acúmulo de células inflamatórias no tecido epitelial, acaba acelerando o desenvolvimento de DK. Complicações cardíacas, como miocardite, ocorrem em mais da metade dos pacientes, sendo diagnosticada pela elevação da troponina I e redução da fração de ejeção do ventrículo esquerdo (SANTOS BS, et al., 2020).

Um estudo realizado em um hospital em Paris, reportou 21 pacientes com média de idade 7.,9 (3.7-16.6) anos, em que 9 tiveram história recente de sintomas virais e um paciente apresentou anosmia, sendo 0 intervalo médio entre esses sintomas e a manifestação dos sintomas de Kawasaki, 45 dias (18-79 dias). 0 contato recente com parentes com sintomas virais foi relatado em 10 famílias, sendo 5 destas altamente suspeitas de covid-19 (apresentavam ageusia, anosmia e achados sugestivos em tomografia computadorizada (TC) de tórax). O resultado de PCR-RT e Anticorpos IgG para SARS-CoV-2 sugeriram relação causal, sendo que o primeiro foi positivo em 8 (38\%) dos 21 pacientes, e o segundo foi positivo em 19 dos 21 (90\%). Sendo 11 (52\%) DK completa e 10 DK incompleta; 10 (48\%) manifestaram derrame pericárdico e 16 (76\%) miocardite com Fração de Ejeção de Ventrículo Esquerdo (FEVE) entre 10\% e 57\% (TOUBIANA J, et al., 2020).

Uma pesquisa feita em centros pediátricos nos Estados Unidos mostrou que a associação de SIM-C com SARS-CoV-2, 131 (70\%) testados positivos para PCR-RT, sorologia ou ambos, leva a casos graves em crianças e adolescentes previamente hígidos, com uma amostra de 186 pacientes e média de idade de 8,3 anos e $62 \%$ masculino, documentou achados de DK em 74 (40\%) e acometimentos multissistêmico como cardiovascular em $80 \%$, sendo 15 pacientes (8\%) documentados com aneurisma de coronária; muco cutâneo $74 \%$ e respiratório $70 \% ; 80 \%$ das crianças precisaram de UTI (FELDSTEIN LR, et al., 2020). 
Conforme reporte de estudo em quatro centros terciários de cuidados em Paris, 20 crianças admitidas por choque e miocardite aguda com FEVE de 35\% (25\%-55\%), troponina $269 \mathrm{ng} / \mathrm{mL}$ (31-4607) e hipotensão arterial com apresentação principalmente vasoplégica, apresentavam pelo menos 1 critério de DK (febre, $n=20$, eritema cutâneo, $n=10$; conjuntivite, $n=6$; quelite, $n=5$; adenite, $n=2$ ) mas nenhuma com a forma completa. PCR para SARS-CoV-2 e Sorologia positivas em 10 e 15 crianças respectivamente. 1 criança teve PCR e sorologia negativas, mas tomografia computadorizada de tórax típica de SARS-CoV-2 (GRIMAUD M, et al., 2020).

Um estudo observacional com 2 grupos, sendo o primeiro composto por 19 pacientes (3.0 anos) realizado entre Janeiro de 2015- Fevereiro de 2020, e o segundo com 10 pacientes (7.5 anos), realizado em Fevereiro até Abril de 2020, ambos em Bergano, Itália, mostrou aumento de 30 vezes na incidência de doenças semelhantes a Kawasaki durante SARS-CoV-2. As crianças diagnosticadas após início da pandemia de Covid-19 apresentavam resposta imunológica ao vírus, eram mais velhas e tinham maior taxa de comprometimento cardíaco (2 de 19 do grupo 1, contra 6 de 10 do grupo 2), quando comparadas as crianças com DK sem associação com Sars-Cov-2 (VERDONI L, et al., 2020).

No grupo 2,80\% apresentaram IgG positivo, sendo que 50\% (5 das 10) apresentou a forma completa de DK. Essas apresentaram conjuntivite não exsudativa, anomalias na mão e pé (eritema ou endurecimento firme, ou ambos) e rash polimórfico. $80 \%$ dos com forma clássica apresentaram também alterações na boca e/ou lábio. Os com DK incompleto, $20 \%$ apresentaram aneurisma de coronária esquerda, redução da fração de ejeção e regurgitação da valva mitral. Além disso, $50 \%$ do grupo 2 apresentou hipotensão e sinais clínicos de hipoperfusão e 8 de 10 apresentaram hiponatremia, aumento da porcentagem de neutrófilos e trombocitopenia. Quando comparados ao grupo 1, esse apresentou predominância da forma clássica de DK (68\%), nenhum paciente apresentou hipotensão, sinais de hipoperfusão e se recuperaram completamente sem aneurisma de artéria coronária residual. Além do que, ecocardiograma anormal foi encontrado em $60 \%$ dos pacientes do grupo 2 versus 10\% do grupo 1 (VERDONI L, et al., 2020).

Son MBF e Friedman K (2020), sugeriram a doença SIM-C como a causa mais provável, visto que apresenta critérios diagnósticos semelhante a DK (idade $<21$ anos; febre $>38^{\circ} \mathrm{C}$, ou subjetiva por $>24 \mathrm{~h} ; 1$ ou mais exames laboratoriais que confirmem inflamação; evidência de doenças clínicas graves em 2 ou mais sistemas; sem outro diagnóstico plausível) além de teste positivo para SARS-COV-2 por RT-PCR, sorologia ou antígeno ou história de exposição nas últimas 4 semanas do início dos sintomas e salientando o fato de nem todos os critérios de DK estarem presentes, porém com epidemiologia mais compatível com os quadros apresentados pós pandemia de COVID-19 (RIPHAGEN S, et al., 2020; VERDONI L, et al., 2020).

De acordo com Riphagen S, et al. (2020), os primeiros estudos sobre a SIM-C foram do Reino Unido em abril de 2020 e logo vieram outros estudos de outras partes do mundo, incluindo Europa, Canadá, Estados Unidos, África do Sul, Ásia, Espanha, entre outros (LICCIARDI F, et al., 2020; VERDONI L, et al., 2020). Esses estudos descrevem crianças com SIM-C preenchendo critérios de DK completa e incompleta, porém a epidemiologia das crianças do estudo diferiram significativamente da DK clássica, afetando crianças mais velhas, com idade média de 8 anos, adolescentes previamente sadios e os de origem hispânica e africana, portanto, contrapondo a epidemiologia da DK, mais comum em descendentes asiáticos (SON MBF e FRIEDMAN K, 2020).

Nesse contexto epidemiológico é importante ressaltar que entre o pico de incidência de COVID-19 e o aparecimento de casos de SIM-C foi de 3 a 4 semanas, relatado em diversos estudos (FELDSTEIN LR, et al., 2020; DUFORT EM, et al., 2020). Segundo Giray T, et al. (2016), uma hipótese que explica esse intervalo é o tempo de desenvolvimento de imunidade adquirida e sugere que a SIM-C possa representar uma complicação pós-infecciosa do vírus, o que coincide com os achados de DK que apresenta características epidemiológicas de sazonalidade e durante epidemias, favorecendo a hipótese de um agente infeccioso causal, geralmente viral, porém em indivíduos geneticamente susceptíveis (MCCRINDLE BW, et al., 2017).

Além disso, é sabido que a DK também tem demonstrado associação com outras formas antigênicas virais, como adenovírus, enterovírus, parvovírus, rhovírus, varicella, Epstein Barr e dengue prévias ao diagnóstico, tendo picos de incidência durante epidemias, primavera e inverno (GIRAY T, et al., 2016; CHANG LY, et al., 
2014). Outros estudos demonstram uma associação entre infecções respiratórias virais e $D K$, variando de $9 \%$ a $42 \%$ dos pacientes com teste positivo para infecção respiratória viral 30 dias antes do diagnóstico de DK (BENSELER SM, et al., 2005).

Outras afecções desencadeadas por infecções virais tais quais linfohistiocitose secundária que tem como característica uma reação inflamatória intensa, assim sendo diagnostico diferencial das síndromes discutidas acima (Tabela 1). Apesar de ser uma síndrome subdiagnosticada tem sua relevância por ser fulminante e fatal, fato esse sendo causado por falhas de múltiplos órgãos. Com a finalidade de reconhecer essa moléstia seus pontos importantes são: febre incessante, citopenia, hiperferritinemia, envolvimento pulmonar, incluindo a síndrome respiratória aguda grave, em $50 \%$ dos pacientes. Um perfil de citocinas interleucina-2, interleucina-7, fator estimulador de granulócitos, interferon gama, proteína 10 induzida por interferon gama, proteína inflamatória de macrófago 1 alfa, fator de necrose tumoral alfa, são preditores de mortalidade, segundo Mehta P (2020).

Tabela 1 - Diagnóstico de linfohistiocitose secundária.

\begin{tabular}{ccccccc}
\hline- & Quesitos & Pontos & Quesitos & Pontos & Quesitos & Pontos \\
\hline Temperatura & $<38,4^{\circ} \mathrm{C}$ & 0 & $38 \cdot 4-39 \cdot 4{ }^{\circ} \mathrm{C}$ & 33 & $>39,4{ }^{\circ} \mathrm{C}$ & 49 \\
\hline Organomegalia & Nenhum & 0 & $\begin{array}{c}\text { Hepatomegalia ou } \\
\text { esplenomegalia }\end{array}$ & 23 & $\begin{array}{c}\text { Hepatomegalia } \\
\text { e } \\
\text { esplenomegalia }\end{array}$ & 38 \\
\hline Citopenias & $\begin{array}{c}\text { Uma } \\
\text { linhagem }\end{array}$ & 0 & Duas linhagens & 24 & Três linhagens & 34 \\
\hline $\begin{array}{c}<1- \\
\text { Triglicerídeo }\end{array}$ & 0 & $1,5-4,0 \mathrm{mmol} / \mathrm{L}$ & 44 & $>4,0 \mathrm{mmol} / \mathrm{L}$ & 64 \\
\hline Fibrinogênio & $>2 \cdot 5 \mathrm{~g} / \mathrm{L}$ & 0 & $\leq 2 \cdot 5 \mathrm{~g} / \mathrm{L}$ & 30 & & 50 \\
\hline $\begin{array}{c}\text { Ferritina } \\
\text { Aspartato }\end{array}$ & $<2000 \mathrm{ng} / \mathrm{ml}$ & 0 & $2.000 \mathrm{a} 6.000 \mathrm{ng} / \mathrm{ml}$ & 35 & $>6000 \mathrm{ng} / \mathrm{ml}$ & 50 \\
$\begin{array}{c}\text { aminotransferase } \\
\text { sérica }\end{array}$ & $<30 \mathrm{IU} / \mathrm{L}$ & 0 & $\geq 30 \mathrm{IU} / \mathrm{L}$ & 19 & - & - \\
\hline $\begin{array}{c}\text { Linhagem } \\
\text { fagocítica }\end{array}$ & não & 0 & $\mathrm{Sim}$ & 35 & - & - \\
\hline Imunossupressão & não & 0 & $\mathrm{Sim}$ & 18 & - & - \\
\hline
\end{tabular}

Fonte: Lessa AR, et al., 2021. Baseado em Mehta P, 2020.

O HScore 11 gera uma probabilidade para a presença de HLH secundário. HScores maiores que 169 são $93 \%$ sensível e $86 \%$ específico para HLH. Observe que a hemofagocitose da medula óssea não é obrigatória para o diagnóstico de HLH. HScores podem ser calculados usando uma calculadora HScore online. $11 \mathrm{HLH}$ = linfo-histiocitose hemofagocítica. * Definido como a concentração de hemoglobina de $9,2 \mathrm{~g} / \mathrm{dL}$ ou menos ( $\leq 5 \cdot 71 \mathrm{mmol} / \mathrm{L}$ ), uma contagem de leucócitos de 5.000 glóbulos brancos por $\mathrm{mm}^{3}$ ou menos, ou contagem de plaquetas de 110000 plaquetas por $\mathrm{mm}^{3}$ ou menos, ou todos esses critérios combinados. † HIV positivo ou recebendo terapia imunossupressora de longo prazo (ou seja, glicocorticóides, ciclosporina, azatioprina).

Tendo em vista a grande morbimortalidade, o diagnóstico precoce, tal qual seu tratamento nos casos de DK identificados, são de extrema importância. O tratamento preconizado pela American Heart Association Guidline, segundo Brain W, et al. (2020) deve ser feito o mais rápido possível com imunoglobulina humana intravenosa além de ácido-acetilsalicílico (AAS), em doses antiagregantes para crianças afim de evitar síndrome de Reye, além de tratamentos adjuvantes caso necessários, como nos casos de alto risco de desenvolvimento de aneurismas coronarianos. Outras alternativas também estão disponíveis para uso em casos específicos.

Considerando as semelhanças entre COVID 19, DK, SIM-C e HLHs, a corticoterapia atua como base terapêutica e a conduta mais efetiva atualmente, porém em alguns casos clínicos em que COVID e outras doenças confluem não houve uma melhora significativa de todos os sinais e sintomas, necessitando do acréscimo de outras entidades terapêuticas. No quadro de DK, já explicitado acima a necessidade da adição 
de imunoglobulinas e AAS, na SIM- C as opções terapêuticas incluem esteróides, imunoglobulina intravenosa, bloqueio seletivo de citocinas (por exemplo, anakinra ou tocilizumab) e inibição de JAK e finalmente HLHs pelo protocolo HLH-2004 em ambiente pediátrico, a primeira linha de tratamento é glicocorticóides e quimioterapia (MEHTA P, 2020).

\section{CONSIDERAÇÕES FINAIS}

A pandemia causada pelo Sars-coV2 é uma questão muito nova para a comunidade médica internacional, que a cada dia descobre coisas novas sobre tal vírus. Em vista do exposto, existem diversos argumentos para a confirmação da relação e sua adição como mais um de uma série de vírus já comprovadamente relacionados com a DK, dentre eles o aumento da sua incidência semanas após picos infecciosos, tanto em sua forma completa quanto incompleta. Contudo também existem diversos questionamentos que trazem dúvidas em afirmar tal relação, como a epidemiologia fora do padrão já conhecido da doença, tendo sido observada uma apresentação mais tardia (8-11 anos) e atingir populações como a afrodescendente e hispânica com mais frequência que os asiáticos, classicamente a população mais acometida. Portanto, conclui-se a necessidade de um tempo maior de observação epidemiológica e avaliação da casuística antes seja possível tirar conclusões definitivas se a SIM-C faz parte do espectro da DK e a epidemiologia divergente encontrada na literatura é secundária a variação étnica ou se realmente seria uma complicação à parte.

\section{REFERÊNCIAS}

1. BERTHELOT JM, et al. Kawasaki-like diseases, and thrombotic coagulopathy in COVID-19: delayed over-activation of the STING pathway? Emerging Microbes \& Infections, 2020; 9: 1514-1520.

2. BRIAN W, et al. Diagnosis, Treatment, and Long-Term Management of Kawasaki Disease: A Scientific Statement for Health Professionals from the American Heart Association. Circulation, 2017: 139: 17.

3. DEVILLE JG, et al. Coronavirus disease 2019 (COVID-19): Clinical manifestations and diagnosis in children. UpToDate, 2020: 36.

4. FELDSTEIN LR, et al. Multisystem Inflammatory Syndrome in U.S. Children and Adolescents, New England Journal of Medicine, 2020; 383: 334-346.

5. FELDMAN SR, et al. Coronavirus disease 2019 (COVID-19): Cutaneous manifestations and issues related to dermatologic care. UpToDate, 2020: 30.

6. FIGUEROA EIR, et al. Incomplete Kawasaki Disease in a Child with COVID-19. Clinical Case Letters, 2020; 57: 680681.

7. GONÇALVES LF, et al. Kawasaki and COVID-19 disease in children: a systematic review. Revista da Associação Médica Brasileira, 2020; 66: 136-142.

8. JONES VG, et al. COVID-19 and Kawasaki Disease: Novel Virus and Novel Case. Hospital Pediatrics, 2020; 10, 537 541.

9. JONES VG, et al. COVID-19 and Kawasaki disease: novel virus and novel case. Hosp. Pediatr, 2020.

10. KLEIN-GITELMAN M, et al. Kawasaki disease: Clinical features and diagnosis. UpToDate, 2020: 23.

11. LICCIARDI F, et al. SARS-CoV-2-Induced Kawasaki-like hyperinflammatory syndrome: a novel COVID phenotype in children. Pediatrics, 2020: 1711.

12. LOOMBA RS, et al. COVID-19 and Kawasaki syndrome: should we really be surprised? Cardiology in the Young, 2020; $1: 1-2$.

13. PANUPATTANAPONG S, BROOKS EB. New spectrum of COVID-19 manifestations in children: Kawasaki-like syndrome and hyperinfl ammatory response. Cleveland Clinic Journal Of Medicine, 2020;1: 1-4.

14. RIVERA-FIGUEROA EI, et al. Incomplete Kawasaki Disease in a Child with Covid-19. Indian Pediatr, 2020.

15. ROWLEY AH, SHULMAN ST. The epidemiology and pathogenesis of Kawasaki disease. Front Pediatr. $2018 ; 6: 374$.

16. SANTOS BS, et al. Clinical-epidemiological relation between SARS-COV-2 and Kawasaki disease: an integrative literature. Revista Paulista de Pediatria,2020; 39: 1-8.

17. SANTOS EH, et al. SARS-CoV-2 infections with emphasis on pediatric patients: a narrative review. Revista do Instituto de Medicina Tropical de Sâo Paulo, 2020: 62: 4-5

18. SON MBF, et al. Coronavirus disease 2019 (COVID-19)-related multisystem inflammatory syndrome in children (MISC): Clinical features, evaluation, and diagnosis. UpToDate, 2020: 29.

19. SON MBF, et al. Coronavirus disease 2019 (COVID-19)-related multisystem inflammatory syndrome in children (MISC): Management and outcome. UpToDate, 2020: 20.

20. TOUBIANA J, et al. Kawasaki-like multisystem inflammatory syndrome in children during the covid-19 pandemic in Paris, France: prospective observational study. BMJ, 2020.

21. VERDONI L, et al. An outbreak of severe Kawasaki-like disease at the Italian epicentre of the SARS-CoV-2 epidemic: an observational cohort study. Lancet, 2020; 395:1771-1778.

22. VINER RM, WHITTAKER E. Kawasaki-like disease: emerging complication during the COVID-19 pandemic. http://www.thelancet.com, 2020; 395: 1741-1743.

23. ZOILO MA, et al. Manifestaciones Extra pulmonares de la infección por SARS-CoV-2 em Pediatría. Revisión. Anales de la Facultad de Ciências Médicas. 2020; 53(2): 87-104. 